\title{
Téoros
}

Revue de recherche en tourisme

\section{Le contrôle de la chaîne de distribution}

\section{Paul Simier}

Volume 9, numéro 3, novembre 1990

La distribution du produit touristique

URI : https://id.erudit.org/iderudit/1079882ar

DOI : https://doi.org/10.7202/1079882ar

Aller au sommaire du numéro

Éditeur(s)

Université du Québec à Montréal

ISSN

0712-8657 (imprimé)

1923-2705 (numérique)

Découvrir la revue

Citer cet article

Simier, P. (1990). Le contrôle de la chaîne de distribution. Téoros, 9(3), 3-6. https://doi.org/10.7202/1079882ar d'utilisation que vous pouvez consulter en ligne.

https://apropos.erudit.org/fr/usagers/politique-dutilisation/ 


\section{Le contrôle de la chaîne de distribution}



Source: Le Journal du Téléphone, no.9

"Tout notre métier dépend de la distribution." Gilbert Trigano, le grand patron de Club Méditerranée $e^{(1)}$, la multinationale des vacances, est catégorique: bien sûr il importe de maitriser un produit touristique qui sache répondre aux besoins des consommateurs, mais il faut aussi savoir le vendre, et bien le vendre. Pour cela il faut contrôler ou du moins savoir stimuler le réseau de distribution. En matière de tourisme, l'importance des réseaux de distribution est d'ailleurs tellement grande qu'il ne se passe pas, depuis quelques années, de saison qui $n$ 'apporte pas, ici comme ailleurs, de bouleversements majeurs dans la façon de mettre le produit touristique en marché.

Plus un pays émetteur de touristes a du poids dans la balance des échanges touristiques, plus il compte $\mathrm{d}^{y}$ agences de voyages, la vitrine la plus visible de tout réseau de distribution.

On compte quelque 50000 agences dans le monde ${ }^{(2)}$. La plupart des agences se situent dans les pays émetteurs de touristes. Les pays européens à eux seuls regroupent ainsi la moitié des agences de la planète. Ultime

\footnotetext{
" Monsieur Paul Simier est chroniqueur en tourisme et
} charge de cours a l'UOAM. maillon de la chaîne de distribution, "l'agence de voyages est un intermédiaire privilégié entre les différents prestataires de services touristiques et les touristes. Elle n'est pas pour autant l'intermédiaire unique et obligét(3).

En fait, le taux de recours aux agences de voyages s'avère en moyenne assez faible. Au Québec, environ le quart des voyageurs fait affaire avec un agent de voyages avant de voyager ${ }^{(4)}$. Dans les autres pays occidentaux, le taux de recours aux agents de voyages varie énormément. Il serait ainsi de 7 à 10 pour cent en France, de 25 pour cent en RFA et aux Pays-Bas et de 85 pour cent au Japon ${ }^{(5)}$.

Le faible taux de recours aux agences de voyages $s^{3}$ explique habituellement par le fait que bien des consommateurs traitent directement avec les fournisseurs de services touristiques (transporteurs, loueurs d'autos, hôtels) dès lors qu'il s'agit d'une destination proche ou de lieux de vacances avec lesquels ils sont déjà familiers.

Ainsi, on constate que les Québécois font rarement appel à un conseiller en voyages pour planifier un voyage au Québec, dans les États américains voisins ou encore en

Floride. Leconsommateur 's'adresse, dans ce genre de situation, directement aux fournisseurs, quand il n'entreprend pas le déplacement par ses propres moyens, avec son autopar exemple, puis en négociantà la pièce les autres services touristiques indispensables.

Les touristes ont plus facilement recours aux agents de voyages dès qu' il s'agit d'une destination plus lointaine et qu' intervientle facteur de la complexité des relations avec les fournisseurs de services. La barrière de la langue, la complexité des réseaux de réservations propres à un pays touristique, la méconnaissance des standards en vigueur à l'étranger sont autant de facteurs qui amènent le touriste à faire préparer son voyage par l'intermédiaire qu'est l'agent de voyages.

Les agences se trouventen effet aujourd'hui équipées pour la plupart de moyens de télécommunications sophistiqués (télex, télécopieur, ordinateur) qui leurpermettent de communiquer facilement avec $1^{\prime}$ ensemble des fournisseurs.

Avant de considérer le rôle des agences de voyages et les tendances qui affectent ce maillon privilégié du réseau de distribution, il importe d'analyser le comportement des fournisseurs de services touristiques qui, de tout temps, ont pratiqué la vente directe et cherché à avoir la mainmise sur le réseau de distribution.

Les sites les plus exceptionnels ne peuvent prétendre attirer des touristes, d'une part si les moyens de transport permettant d'y accéder ne sont pas disponibles, d'autre part si les modes d'hébergementne sont pas développés.

\section{La maîtrise du transport et de l'hébergement}

Ce postulat établi, on peut énoncer le principe sur lequel est fondé tout réseau de distribution: qui maitrise le transport et l'hébergement est en mesure d'influer fortement sur le système de distribution et mềme de le contrôler.

Quels que soient le type de voyage et la motivation du touriste - voyage de 
découverte, de repos-farniente, sportif, culturel ou autres -, les deux éléments clés du produit touristique demeurent le transport et l'hébergement. $\AA$ la base du réseau de distribution se situent donc les compagnies de transportet les entreprises d'hébergement et de restauration.

Traditionnellement, la plupart de ces entreprises dont les services constituent la base même du produit touristique disposent de leur propre canal de distribution direct ${ }^{(6)}$, Les compagnies aériennes régulières, dont la mission consiste à assurer des vols à horaires fixes toute l'année, organisent, mettent en marché et vendent leurs propres produits. De plus, - on le verra ensuite elles ont recours à un canal de distribution indirect impliquant des intermédiaires.

Depuis quelques années, le service de transport aérien régulier revêt diverses formes selon les types de voyageurs visés. Plus ou moins élaboré selon les contraintes du passager, plus ou moins coûteux selon les contraintes et restrictions qui y sont rattachées, le service de transport aérien est multiple et commande dès lors l'utilisation de canaux de distribution variés.

La plupart des avions assurant des vols réguliers comportent aujourd'hui deux sinon trois classes (Économique, Affaires, Première). Celles-ci se distinguent selon le confort (qualité de fauteuil, écartement des rangées, espace, tranquillité) mais aussi la qualité du service (repas, petites attentions à bord, conditions de réservation, d'enregistrement et de paiement).

Les compagnies aériennes régulières exploitenten premier lieu plusieurs moyens de vente directe.

Le premier est la centrale de réservations. Les préposés, des agents de réservations à la fois conseillers et vendeurs, répondent aux appels téléphoniques du public et concrétisent les réservations. Aux bureaux de vente ayant pignon sur rue dans les centres urbains ou dans les aéroports, les voyageurs peuvent également traiter directement avec des vendeurs de la compagnie de transport. De plus, avec le développement des voyages d'affaires, les compagnies aériennes ont installé des terminaux d'ordinateurs au sein des entreprises commerciales clientes.

La vulgarisation de la télématique (Minitel, Alex, Vidéoway, etc.) met un autre outil de vente directe à la portée des fournisseurs de services touristiques pour communiquer directement avec les consommateurs. Ces systèmes, relativement nouveaux au Québec, sont déjà largement utilisés dans d'autres pays par les grands transporteurs surtout (mais aussi les hôteliers et les autres fournisseurs). Ces systèmes ont la particularité d'être interactifs, soit de permettre au consommateur de passer directement des ordres et des commandes.

Le premier marché qu'exploitent les compagnies aériennes régulières, c'est évidemment celui des voyages d'affaires. La recette par passager payant le plein tarif représente en effet entre quatre et six fois celle d'un billet d'avion destiné au vacancier. Et pourtant, tous ces passagers se côtoient, ou presque, à bord.

Ces différences notables de tarifs s'expliquent selon le principe: plus le prix est bas, plus le billet d'avion comporte des restrictions (date limite de réservation avant le départ, conditions de paiement, durée minimale et maximale deséjour, conditions de remboursement, pénalités en cas de changement des dates de voyage, etc.).

Les entreprises hôtelières ont en quelque sorte calqué leurs services sur ceux des compagnies aériennes en développant des produits spécifiques à l'intention des gens d'affaires. Elles participent d'ailleurs conjointement, notamment à travers les plans de récompense pour voyageurs assidus, à la mise en marché des services touristiques destinés aux gens d'affaires.

Tout comme le font les compagnies aériennes, les réseaux hôteliers exploitent pour la plupart un réseau direct de distribution. Ainsi, les groupes hôteliers possèdent leur propre centrale de réservations, accessible aux consommateurs. Ils sont tout aussi présents que les grands transporteurs dans les systèmes télé̉matiques.

$\mathrm{L}$ 'interaction des compagnies intervenant dans le transport (notamment aérien) et celles offrant l'hébergement est d'ailleurs tellement forte que les mêmes groupes contrôlent de plus en plus l'une et l'autre de ces composantes de base du produit touristique. SAS, KLM, Air France, Swissair, Lufthansa, Japan Air Lines et nombre de compagnies américaines possèdent leur propre filiale hôtelière ${ }^{(7)}$. Le groupe financierCanadien Pacifique a ainsi contrôlé simultanément CP Air (rachetée depuis par PWA) et CP Hôtels. Le groupe $\mathrm{CN}$, avec CNRailet les hôtels $\mathrm{CN}$, constitue un autre exemple du genre.

Groupe A, en France, financé notamment par le puissant GMF (Groupedes mutuelles des fonctionnaires), contrôle pour sa part l'hébergement (Club Aquarius), le transport aérien (Air Liberté) ainsi que des réseaux. de distribution.

\section{Concentration: transport et hôtellerie}

Les deux réseaux de distribution directs (transports et hôtellerie) s'appuient ainsi mutuellement. L'intégration de ces deux composantes du produit touristique est si poussée que l'on peut, en appelant la compagnie de transport aérien, faire réserver des chambres d'hôtel, évidemment dans le réseau intégré au même groupe.

L'intégration des services offerts aux voyageurs s'avère de plus en plus poussée. Plusieurs hôtels ainsi intégrés deviennent des points de représentation de la compagnie aérienne soeur. Le voyageur se voit ainsi offrir, à la réservation de l'hôtel, la possibilité de faire enregistrer ses bagages et de se faire délivrer sa carte d'embarquement à bord d'un vol de la compagnie aérienne du même groupe.

\section{Le réseau indirect}

Aussi tentaculaires que puissent parfois être les réseaux de distribution directs mis en place par les compagnies aériennes régulières et les réseaux hôtelierș, ces fournisseurs font aussi appel à un réseau de distribution indirect.

Ces sociétés mettent à profit le réseau constitué par les agences de voyages pour mieux vendre d'abord ce qui pour elles est le plus rentable: les voyages et séjours de gens d'affaires. C'est ainsi que toutes les agences de voyages sont normalement en mesure de proposer à leurs clients les services de tous les transporteurs comme ceux de tous les autres fournisseurs de services touristiques.

La baisse des voyages d'affaires durant certaines saisons a amené depuis longtemps les transporteurs aériens à s'intéresser aux vacanciers pourrentabiliser leurs vols. $C^{\prime}$ est ainsiquel'on voit apparaitre les tarifs réduits de billets d'avion et de chambres d'hôtel quand les voyageurs d'affaires se montrent moins nombreux.

C'est à ce niveau qu'intervient le voyagiste, encore dénommé grossiste en voyages ou tour-opérateur. Intermédiaire entre, d'une part, le transporteur et l'hôtelier, et, d'autre part, l'agence de voyages, le voyagiste a pour rôle d'organiser le produit touristique en en assemblant les diverses composantes: transport, hébergement, restauration, assistance, activités. C'est ce que l'on appelle le voyage à forfait.

Le transporteur aérien confie au voyagiste des blocs de sièges ou affrète même des avions complets, tandis que les entreprises 
hôtelières signent avec lui des contrats de commercialisation de chambres. Pour garder la mainmise sur la distribution, les grands transporteurs se sont d'ailleurs depuis longtemps dotés de filiales voyagistes $^{(s)}$. Ces intermédiaires, selon la tradition des différents pays, peuvent posséder leurs propres bureaux de vente dans les grands centres urbains et se faire représenter de plus par l'ensembledu réseau d'agences détaillantes, ou encore éviter, comme au Québec, toute vente directe pour ne pas indisposer les détaillants.

\section{Transporteurs à la demande et intégration}

Parallèlement aux compagnies aériennes régulières, on a vu proliférer depuis vingt ans les transporteurs à la demande (charters) dont la particularité est de n'assurer des vols (généralementoccasionnels) que s'ily a contrat de location de la part d'un voyagiste.

Les transporteurs qui assurent des vols nolisés interviennent d'abord sur des destinations qui ne sont pas desservies par des vols réguliers ou encore en concurrence avec des vols réguliers sur des destinations où le potentiel de clients est important. La compagnie de charters ne pratique donc aucune vente directe et ne possède pas de service de réservations. Dans ce cas, le voyagiste devient maître du produit transport et responsable de sa distribution à travers le réseau des agences de voyages.

L'histoire de la dernière décennie a démontré que plus les compagnies aériennes régulières allaient de l'avant dans l'intégration de leurs activités et de leurs réseaux de distribution (transport, hôtellerie, voyagiste), plus les transporteurs à la demande se multipliaient par ailleurs.

Pour les voyagistes indépendants, il $s$ 'agissait en effet de se donner les moyens à la fois de se différencier dans le produit et d'échapper à la mainmise des transporteurs réguliers. C'est ainsi que dans bien des paysoccidentaux, àl' initiative devoyagistes soucieux d'intégrer verticalement leurs activités, sont nés des transporteurs charters, et réciproquement d'ailleurs des compagnies charters ont cré leurs propres filiales voyagistes ${ }^{(9)}$.

\section{L'agent de voyages: I'ultime maillon}

Les agences de voyages, malgré l'existence d'un réseau direct contrôlé par les transporteurs et les hôteliers, demeurent l'ultime maillon et le plus polyvalent dans la distribution du produit touristique. Leur nombre considérable, leur implantation partout où se retrouve la population, leur mode de rémunération sont autant de facteurs qui expliquent que tous les fournisseurs utilisent les agences comme des vitrines.

L'agent de voyages est en effet rémunéré seulement à commission par ses fournisseurs. (Dans certains pays d'Europe, l'agent de voyages peut facturer à ses clients des frais forfaitaires de dossier.) Il est ainsi le représentant de toutes les compagnies de transport régulières, des réseaux hốteliers, des autres fournisseurs de services et des voyagistes.

Tel est donc le schéma général du réseau de distribution, qui trouve son application dans la plupartdes pays occidentaux. L'évolution du réseau de distribution en place au Québec mérite d'être analysée à ce stade car elle illustre les phénomènes d'intégration des fonctions, de regroupement des agences et de spécialisation.

\section{Au Québec: regroupements et intégration}

Le réseau de distribution québécois est engagé depuis dix ans dans le même processus que celui qui caractérise les autres réseaux de par le monde. Il s'agit d'un renforcement du rôle des chaînes et des regroupements d'agences de voyages détaillantes etd'une tendance à l'intégration verticale des activités allant même parfois de la production du service touristique jusqu'à la vente au détail.

Dans le même temps, l'informatique sous toutes ses formess'estdéveloppée tant dans le réseau des intermédiaires que dans le circuit de vente directe. Il y a un peu moins de dix ans, l'on a d'abord vu les compagnies aériennes régulières développer, sous forme de filiales, la fonction voyagiste. Des exemples: AirCanada développait Touram alors que les défuntes CP Air, Nordair, Quebecair, Wardair eurent aussitoutes leurs filiales voyagistes. Aujourd'hui, Air Canada et Canadien International traitent directement par leurs filiales respectives, Touram et Vacances Canadien, la fonction voyagiste. Ces voyagistes ont toujours distribué leurs voyages à forfait par le réseau des agences détaillantes et ont même soigneusement évité de pratiquer la vente directe.

Mais les transporteurs, les compagnies mères des voyagistes intégrés, se trouvent facilement en conflit d'intérêts dès qu'il s'agit d'affréter des blocs de sièges aux voyagistes indépendants, des concurrents directs, qui arguent le manque d'étanchéité entre le transporteur et sa filiale voyagiste. Dans le même temps que se pratiquaitcette intégration des fonctions transport et voyagiste chez les transporteurs réguliers, se créaient des transporteurs charters indépendants vers lesquels se sont portés évidemment les voyagistes indépendants.

Cela se passait dans les années quatrevingt. Deux clans se sont ainsi crés.s. Durant quelques années, le produit offert se différenciait par la qualité du service (avions gros-porteurs notamment) offerte par les transporteurs réguliers, alors que les derniers nés des transporteurs à la demande (Nationair, Quebecair et Minerve Canada) exploitaient des avions de moindre capacité, plus désuets et moins confortables.

Avec des coûts d'exploitation totalement différents (personnel plus limité, investissements moins lourds, plus grande flexibilité), ces transporteurs charters pouvaient offrir des prix alléchants à leurs clients voyagistes Les transporteurs à la demande et leurs clients, les voyagistes indépendants, se sont ainsi renforcés, se distinguant du coup des groupes intégrés.

Ce phénomène n'a pas manqué épisodiquement de déclencherde sanglantes guerres de prix, notamment chaque fois qu'un nouveau transporteur charter cherchait à se faire une place au soleil. Cela a entraîné des faillites ou du moins des rachats. Mais chaque fois, un autre transporteur est né peu de temps après. Après la privatisation de Quebecair et la vente des appareils destinés au nolisement appartenant à cette compagnie, 1 'on a ainsi vu se créer Air Transat, à l'initiative des dirigeants d'un voyagiste.

Groupe Transat (c'était la première fois dans le réseau de distribution que l'on avait recours à une émission d'actions pour créer une compagnie publique inscrite en Bourse), dès sa création, intégrait les fonctions transport (Air Transat), voyagiste (Trafic Voyages), réceptif (vente en Europe par Trafic France et le département réceptif à Montréal).

Peude temps après, Groupe Transat mettait le pied dans la distribution en intégrant partiellement le réseau Consultour-Club Voyages, puis complètement le réseau des agences Tourbec (qui avait parmi ses actionnaires les dirigeants de Trafic Voyages et fondateurs de Groupe Transat) et aussi en rachetant successivement trois voyagistes (Multitour, Maximum Tours (Toronto) et Fantasia). Dans les projets à court terme du groupe figure le contrôle de maillons hôteliers en gestion exclusive. En intégrant deux réseaux de détaillants 
(qui représentent plus du quart des ventes de voyages au détail au Québec), Groupe Transatse donne les moyens de mettre ainsi prioritairementen marché les forfaits de ses voyagistes et de son transporteur.

Pendant ce temps, Nationair, dont les responsables ont toujours proclamé: " $\mathrm{A}$ chacun son métier", continue de n'assurer que la fonction transport, en affrétant ses avions à des voyagistes indépendants.

$\mathrm{L}$ 'an dernier, l'on a vu un groupe torontois intégré (transportet voyagiste) prendre une forte participation dans un voyagiste québécois (Sol-Vac) puis, plus tard, mettre la main sur le réseau de détaillants Constellation. Une forme de répétition de la démarche de Groupe Transat.

Pource qui est de la distribution du voyage a forfait, tout s'articule donc d'abord et avant tout autour du maillon de la chaîne qui maîtrise le transport. L'écart, en matière de service, entre les compagnies régulières et les compagnies de nolisement s'estrétréci au cours des dernières années, ces dernières s'étant dotées de flottes plus récentes et d'avions plus confortables.

La maitrise en bout de ligne du réseau de distribution n'en devient donc que plus importante.

\section{Regroupements et agences spécialisées}

Prendre le contrôle le plus poussé possible des points de détail devient primordial pour les producteurs dans l'industrie du voyage. Avec la concurrence féroce, les marges de profit s"amenuisent. Reste donc le volume.

Les regroupements (Club Voyages et associés. Associatour) et les chaînes d'agences de voyages intégrées (American Express, Thomas Cook, Sears, Marlin, Travelaide, Uniglobe, Constellation, etc.) sesontmultiplies etconsolidés. Ces groupes d'agences sélectionnent généralement quelques fournisseurs privilégiés parmi les transporteurs (pour la billetterie) et les voyagistes (pour les forfaits). Entrent en ligne de compte la qualité du produit même mais aussi celle du service offert aux détaillants pour les fournisseurs. De plus, ces unions permettent aux détaillants d'obtenir de meilleures commissions. II n'est pas rare que sur la base de telles ententes commerciales, des groupes d'agences arrivent maintenant à bénéficier de prix de vente exclusifs alors que $l^{*}$ ensemble du réseau détaillant propose les mềmes forfaits.
Le vacancier moyen a tendance à acheter un prix plutót que l'assurance d'effectuer un voyage de qualité, simple déformation sans doute dans une société de consommation où priment les étiquettes de "spéciaux". Dès lors, les agences qui ne sont pas en mesure aujourd"hui d'afficher des prix compétitifs éprouvent facilement des difficultes.

Dans le même temps qu'ils mettent de l'avant des tarifs attrayants, l'image et la promotion, les regroupements d'agences misenten effet sur la formation du personnel. Est-ce à dire que les seules agences qui surnagent dans cet univers fait d intégrations et de regroupements appartiennent ainsi à des groupes? Certes non. Bien des agences indépendantes ont, dans le même temps, su développer une vocation particulière pour offrir à une clientèle-cible des voyages thématiques.

Misant sur des besoins non pas nouveaux mais nouvellement apparus, ces agences travaillent de manière plus personnalisée et, plutôt que le très grand nombre declients, visent un service de qualité, quitte à en faire payer le prix. Les plus innovatrices dans l'une et l'autre de ces catégories d'agences ont naturellement investi les premières dans la télématique. Consultour-Club Voyages se retrouve ainsidans le système Vidéoway (Vidéotron), tandis que la chaine des agences Funtastique figure parmi les fournisseurs d'Alex (Bell Canada).

Ces groupes d'agences détaillantes ont en effet compris qu'il leur faut contrôler ces moyens de distribution directs plutôtque de laisser leurs propres fournisseurs le faire. Mais, étant donné la timide percée de ces systèmes, la télématique, pourtant porteuse d'avenir, ne suscite pas, pour l'heure, de grands enjeux dans l' intégration des moyens de distribution.

\section{Mondialisation et marché restreint}

Les tendances a l'intégration verticale des réseaux de distribution se confirment dans tous les pays occidentaux émetteurs de touristes. Le principal défi pour l'industrie québécoise du voyage consiste maintenant à s'intégrer dans le mouvement de mondialisation des réseaux.

La dimension très limitée du marché québécois oblige les professionnels, s'ils veulent se donner des chances d'expansion, à envisager plus globalement leurs interventions. Ainsi, alors que bien des voyagistes n'avaient jusqu'a ces derniers temps envisagé que la facette émettrice du tourisme, de plus en plus on les voit développer $l^{\prime}$ aspect réceptif.
Cette tendance est d'autant plus évidente d'ailleurs qu'il s'agit de groupes intégrés qui tentent ainsi de rentabiliser leurs opérations sous deux angles: affrètement d'avions puis opération voyagiste et commercialisation d' un produit touristique complet aux deux extrémités.

D'autres voyagistes, pour offrir plus que les destinations que tous leurs concurrents offrent aussi, ont entrepris de s'allier avec des partenaires dans d'autres pays francophones, notamment en France, pour ainsi partager leurs ressources dans la mise en marché de leurs produits communs. Ilen est ainsi de Tours Chanteclerc allié avec Voyages Hervouet, autocariste, voyagiste et agent de voyages détaillant. Tours MontRoyal, de son cốté, a contracté une entente avec le groupe français Chorus. Air CanadaTouram a créé Touram France en s'alliant à une importante agence réceptive de ce pays.

Tout ceci pose aussi le problème, maintes fois soulevé par la profession, de la formation et du perfectionnement du personnel. Bien des responsables de compagnies dans les réseaux de distribution sont des autodidactes en matière de tourisme,

Pour beaucoup se pose l'épineuse question de la relève dans un milieu où les affaires se font beaucoup en fonction des individus mais qui doit de plus en plus composer avec les règles du grandicommerce international.

Bibllographie at róforences

\begin{abstract}
a) Lives
SERVAN-SCHAEIBER, Jean-Louis, Le métier de patran, Paris, Fayard, 1990, 478 pages. (1) p. 465.

PASOUALINI, J.P. et JACQUOT, B, Tourismes, Paris, Dunod, 1999, 321 pages. (2) p. 44: (3) et (5) p. 46; (18) pp. $280-281$ et 191 p. 293
\end{abstract}

WACHERMANN, Gabriel, Le tourisme International Paris, Armand Colin, 1969, 200 piges. (6) p. 93.

CAZES, G. Le tourisme international - Mirage ou atratégie d'awenlr?, Paris, Hatier, 196 pages. (7) pp. $132-133$

et aussi:

SAMPSON, Anthany, Les empires du clal, Montréal, Boreal, 1996, 332 pages.

SECOR, Montribal, 1982, Rapport sur la fréquentation des agences de voyages (4)

b) Articles de journaux et periodiques

SIMIER, Paul, Le Journal de Montrial, chronique Voyageurs avertis. 1981 a 1990.

Tourisme Plus, Montred, collection 1980 a 1990

Bulletin Voyages, Montreal, collection. 